Keywords: CDDP; oesophageal squamous cell carcinoma; glypican-1; chemoresistance; prognostic predictive biomarker; cis-Diammineplatinum (II) dichloride; isobaric tags for relative and absolute quantitation

\title{
Overexpression of glypican-1 implicates poor prognosis and their chemoresistance in oesophageal squamous cell carcinoma
}

\author{
Hisashi Hara ${ }^{1,2}$, Tsuyoshi Takahashi ${ }^{* 1,2}$, Satoshi Serada², Minoru Fujimoto², Tomoharu Ohkawara²,
} Rie Nakatsuka ${ }^{1}$, Emi Harada ${ }^{2}$, Takahiko Nishigaki ${ }^{1}{ }^{2}$, Yusuke Takahashi ${ }^{2}$, Satoshi Nojima ${ }^{3}$, Yasuhiro Miyazaki ${ }^{1}$, Tomoki Makino ${ }^{1}$, Yukinori Kurokawa ${ }^{1}$, Makoto Yamasaki ${ }^{1}$, Hiroshi Miyata ${ }^{1}$, Kiyokazu Nakajima ${ }^{1}$, Shuji Takiguchi ${ }^{1}$, Eiichi Morii ${ }^{3}$, Masaki Mori ${ }^{1}$, Yuichiro Doki ${ }^{1}$ and Tetsuji Naka ${ }^{*, 2}$

${ }^{1}$ Department of Gastroenterological Surgery, Osaka University Graduate School of Medicine, 2-2 Yamadaoka, Suita, Osaka 565-0871, Japan; ${ }^{2}$ Laboratory of Immune Signal, National Institute of Biomedical Innovation, Health and Nutrition, 7-6-8 Saito-Asagi, Ibaraki, Osaka 567-0085, Japan and ${ }^{3}$ Department of Pathology, Osaka University Graduate School of Medicine, 2-2 Yamadaoka, Suita, Osaka 565-0871, Japan

Background: Despite the recent improvements in multimodal therapies for oesophageal squamous cell carcinoma (ESCC), the prognosis remains poor. The identification of suitable biomarkers for predicting the prognosis and chemo-sensitivity is required to develop targeted treatments and improve treatment results.

Methods: Proteins highly expressed in ESCC cell lines compared with normal oesophageal cell lines were screened by isobaric tag for relative and absolute quantitation (iTRAQ). We identified glypican-1 (GPC1) as a novel molecule. The clinicopathological characteristics of GPC1 were evaluated by immunohistochemistry using ESCC specimens, and clinical parameters were assessed. The correlation between GPC1 expression levels and chemo-sensitivity were analysed in vitro.

Results: In the immunohistochemical assessment of 175 ESCC patients, 98.8\% expressed GPC1. These patients demonstrated significantly poorer prognosis compared with patients with low-GPC1 expression by survival assay $(P<0.001)$. Higher chemoresistance was observed in the GPC1 high-expression group. GPC1 expression levels positively correlated with chemo-sensitivity against cis-Diammineplatinum (II) dichloride (CDDP), and are potentially associated with anti-apoptotic function based on alterations in the MAPK downstream signalling pathway and Bcl-2 family member proteins.

Conclusions: GPC1 is an independent prognostic factor in ESCC and is a critical molecule for altering the threshold of chemoresistance to CDDP.

Oesophageal squamous cell carcinoma (ESCC) is still one of the most refractory cancers in the world (Rustgi and El-Serag, 2014). Although various multidisciplinary therapies improve curability, outcomes are still poor. The underlying reasons for this include surgical complexity, predisposition to lymphatic metastases, as well as the inadequacy of current treatment modalities (Pennathur et al, 2013). For ESCC, cis-Diammineplatinum (II) dichloride (CDDP), and 5-fluorouracil (5-FU) are the commonly used chemotherapeutic agents, with taxane use increasing (docetaxel (DTX) and paclitaxel). Following the completion of clinical trials to evaluate best practices for treating ESCC, neoadjuvant chemotherapy (Hirao et al, 2011; Zheng et al, 2015) and surgery emerged as the recommended treatment strategies (Fujita, 2015). However, the paucity of available treatment agents led us to prioritise the search

*Correspondence: Dr T Takahashi; E-mail: ttakahashi2@gesurg.med.osaka-u.ac.jp or Professor T Naka; E-mail: tnaka@nibiohn.go.jp

Received 9 February 2016; revised 11 May 2016; accepted 17 May 2016; published online 16 June 2016

(c) 2016 Cancer Research UK. All rights reserved 0007-0920/16 
for novel biomarkers with which to predict prognosis and chemosensitivity (Okumura et al, 2014; Karimata et al, 2015).

Advances in proteomics technologies have enabled us to identify unique proteins that distinguish cancer cells from normal cells. Recently an innovative multiplexed quantitative proteomic technology called isobaric tag for relative and absolute quantification (iTRAQ) has been successfully used to detect disease-related proteins. In addition to identifying proteins in samples, this novel technology can also compare relative expression (Ross et al, 2004). Furthermore, the method allows for enrichment of cell-surface proteins via their biotinylation and affinity purification, which enabled us to focus primarily on cell-surface proteins. We have previously reported the effectiveness of the combination of a biotinylation-based approach for the enrichment of cell-surface membrane proteins and iTRAQ in identifying cancer antigens (Yokoyama et al, 2013). In this study, we screened for novel ESCC antigens using a quantitative proteomic approach focused on cellsurface membrane proteins enriched from normal oesophageal epithelial and ESCC cells, and identified overexpression of glypican-1 (GPC1) as an ESCC biomarker.

GPC1 is a cell-surface heparan sulphate proteoglycan (HSPG), which acts as a co-receptor for heparin binding of mitogenic growth factors. It plays diverse roles in cell growth, and adhesion. GPC1 has previously been reported to be overexpressed in breast and pancreatic cancer (Matsuda et al, 2001; Duan et al, 2013; Okolicsanyi et al, 2014; Melo et al, 2015). GPC1 expression has also been linked with malignant progression (e.g., angiogenesis, metastatic potential, and cell-cycle promotion) in cancer cells and is a prognostic factor in that lesion (Kleeff et al, 1998, 1999; Ding et al, 2005; Aikawa et al, 2008). Despite these findings, there have been, to date, no reports to correlate the expression of GPC1 expression and prognosis for ESCC patients.

The aim of this study was to evaluate GPC1 expression in human ESCC specimens and to validate the correlation between GPC1 overexpression and clinicopathological characteristics. In addition, we investigated the mechanism of GPC1-mediated drug resistance to chemotherapeutic agents in ESCC cells, a relationship that was discovered in this study.

\section{MATERIALS AND METHODS}

Cell lines. The human oesophageal epithelial cell 'HEEpiC' was obtained from ScienCell (San Diego, CA, USA) and cultured in Epithelial Cell Medium-2. Human oesophageal epithelial cells 'Het$1 \mathrm{~A}^{\prime}$ were obtained from American Type Culture Collection (ATCC, Rockville, MD, USA) and cultured in complete growth medium (Bronchial Epithelial Cell Medium (BEGM BulleKit)). Nine human oesophageal squamous cancer cell lines (TE-1, TE-5, TE-6, TE-8, TE-9, TE-10, TE-11, TE-14 and TE-15) were obtained from the RIKEN BioResource Center and a human lung squamous cancer cell line (LK-2) was obtained from the Japanese Collection of Research Bioresources (Osaka, Japan), these 10 cell lines were maintained in RPMI 1640 medium. All mediums were supplemented with $10 \%$ foetal bovine serum (FBS; Serum Source International, Charlotte, NC, USA) and $1 \%$ penicillin/streptomycin (Nacalai Tesque, Kyoto, Japan). Cultures were maintained at $37^{\circ} \mathrm{C}$ in a humidified atmosphere at $5 \% \mathrm{CO}_{2}$. The identity of each cell line was confirmed by DNA fingerprinting via short tandem repeat profiling, as previously described (Ferlay et al, 2015).

Capture of cell-surface proteins. Normal oesophageal epithelial cell lines (HEEpiC and Het-1A cells) and six oesophageal cancer cell lines (TE-1, TE-6, TE-8, TE-9, TE-10 and TE-14) were grown to $90 \%$ confluency in three $150-\mathrm{mm}$ dishes, and cell-surface proteins were biotinylated using sulfo-NHS-SS-biotin (Pierce
Chemical, Rockford, IL, USA), as previously described (Yokoyama et al, 2013).

iTRAQ labelling. Samples were labelled with the iTRAQ reagent, according to the manufacturer's protocol (AB SCIEX, Foster City, CA, USA). HEEpiC cells were labelled with iTRAQ reagent 113, Het-1A cells with 114, TE-1 cells with 115, TE-6 cells with 116, TE8 cells with 117 , TE-9 cells with 118 , TE-10 cells with 119 and TE14 cells with 121. Labelled peptide samples were then pooled, desalted and fractionated with strong cation exchange chromatography, as previously described (Yokoyama et al, 2013).

Mass spectrometric analysis. Nano-LC-MS/MS analyses were performed on an LTQ-Orbitrap XL (Thermo Fisher Scientific, Waltham, MA, USA) as previously described (Yokoyama et al, 2013).

iTRAQ data analysis. Protein identification and quantification for iTRAQ analysis were performed using the Proteome Discoverer software (v. 1.3; Thermo Fisher Scientific) and the SwissProt protein database (SwissProt_2012_06, 536489 entries). Taxonomy was set to Homo sapiens (20312 entries) or mammalian (65999 entries). Search parameters were set as previously described (Yokoyama et al, 2013). The raw data from mass spectrometry and peptide identifications were uploaded to PeptideAtlas, available at http://www.peptideatlas.org/PASS/PASS00547. To determine subcellular localisation, all proteins identified in this analysis were analysed using UniprotKB (http://www.uniprot.org/).

Patients and biopsy material. ESCC tissue was obtained from 175 patients who underwent oesophagectomy at the Department of Gastroenterological Surgery, Osaka University Hospital, from 2001 to 2013; the median duration of follow-up was 39 months (range 1-150 months). Informed consent was obtained from all donors, and all studies involving human subjects were approved by the Institutional Review Boards No. 15224 of Osaka University Hospital, and the National Institute of Biomedical Innovation, Health and Nutrition (No. 94). Board-certified pathologists confirmed the diagnoses of all tumours as ESCC following histologic review. TNM 7th edition (Union for International Cancer Control (UICC)) criteria were used to categorise histologic differentiation, as well as the surgical and pathological staging.

IHC analyses. Sections were prepared from formalin-fixed, paraffin-embedded tissue sections $(3 \mu \mathrm{m})$. These were deparaffinized with xylene, then rehydrated in four graded alcohols $(70,80$, 90 and 100\%). Immunohistochemical staining (IHC) for GPC1 was performed using a rabbit polyclonal anti-GPC1 antibody (Atlas Antibodies AB, Stockholm, Sweden, 1:400), with visualisation using Envision ChemMate (Dako, Glostrup, Denmark), according to the manufacturer's protocol. Three independent gastroenterological oncologists (TT, RN and $\mathrm{HH}$ ), blinded to the histologic data, analysed the stained sections, which were also photographed using phase contrast light microscopy (DM2500 with Leica Application Sweat version 3.80; Leica Microsystems GmbH, Wetzlar, Germany).

We defined the expression of GPC1 protein as positive in cancerous cells when their expression of this antigen was greater than that seen in the basal layer, which we used as an internal control (Hewitt et al, 2014). Immunostaining was scored according to the intensity of the staining: 0 , no staining; 1 , normal staining (i.e., the same degree as the basal layer); 2 , strong staining (clearly in excess of that seen in 1). The 'density' of staining (termed the positivity score) was as follows: 0 , denotes $0-9 \%$ positivity; 1 , indicates $10-40 \%$; 2 , indicates $41-70 \%$; and $3,71-100 \%$ positivity. The final IHC score was determined by multiplying the intensity score by the positivity score, resulting in a maximum possible score of 6. These data were referred to as the GPC1 score 
(Yokoyama et al, 2013). Furthermore, we divided patients into two-equally balanced groups by score.

In this study, the Japanese classification of gastric carcinoma, 3rd English edition, was used to categorise pathologic findings for histological response to neoadjuvant chemotherapy (Japanese Gastric Cancer Association, 2011). Grades 0 and 1a were classified as poor histological response groups, whereas grades $1 \mathrm{~b}$ and 2 were classified as good histological response groups.

Establishment of GPC1-knockout and over-expressing cells. For the GPC1 knock out, the TE-14 cell line was used in conjunction with the Crisper-Cas9 system. All-in-one, ready-to-use Cas9 and gRNA expression plasmids targeting human GPC1 (Tarteg ID: HS0000251842) or CRISPR negative control1 (CRISPR06) was used according to the manufacturer's instruction (Sigma-Aldrich, St Louis, MO, USA). Two knock out cell lines termed GPC1 negative-1 and -2 (GN1, GN2), and one mock knock out cell line (as a control), named GPC1 positive (GP-C), were established.

Total cellular RNA was extracted from cell pellets harvested from each cell line using the RNeasy Mini Kit (Qiagen, Hilden, Germany). cDNA was synthesised from total RNA using the QuantiTect Reverse Transcription Kit (Qiagen). To construct a GPC1-expressing vector, the cDNA of GPC1 was amplified by PCR using KOD-plus (Toyobo Co. Ltd., Osaka, Japan) and the following primers: GPC1 forward primer 5 -CGGCCCCGCCATG GAGCTCC- $3^{\prime}$, and GPC1 reverse primer 5'-GGCAGTTACCGC CACCGGGG-3'. The amplified cDNA was inserted into pcDNA3.1/V5-His TOPO TA (Life technologies, Carlsbad, CA, USA) and designated pcDNA3.1-GPC1. The DNA sequence was confirmed using the ABI PRISM 3130XL Genetic Analyzer (Applied Biosystems, Foster City, CA, USA).

To generate a stable GPC1-expressing cell line, LK-2 cells were transfected with pcDNA3.1-GPC1 using Lipofectamine 2000 (Life technologies). Transfected cells were selected using $400 \mu \mathrm{g} \mathrm{ml}^{-1}$ G418 (Life technologies), and thereafter maintained in $200 \mu \mathrm{g} \mathrm{ml}^{-1}$ G418. GPC1-expressing cell lines were established and designated as LK-2 G56 and G57. An LK-2 control cell line, designated LK-2 E29, was also established, with stable transfection accomplished using an empty vector. GPC1 expression of transfected cells was assessed by fluorescence-activated cell sorting (FACS) and western blotting.

GPC1 expression assay. Transfected cells were grown to $80 \%$ confluency in 100-mm dishes. Cells were washed twice in PBS (Nacalai Tesque) and detached in $0.02 \%$ EDTA solution (Nacalai Tesque). Cells were washed twice with cold FACS buffer (PBS supplemented with $1 \%$ FBS and $0.1 \%$ sodium azide), and then incubated with goat anti-GPC1 antibody (R\&D Systems Inc., Minneapolis, MN, USA) at a 1:100 dilution and labelled with PE-labelled donkey $\mathrm{F}\left(\mathrm{ab}^{\prime}\right) 2$ anti-goat IgG antibody (Rockland Inc., Gilbertsville, PA, USA). Stained cells were analysed using a FACS Canto II cytometer (Becton Dickinson, Mountain View, CA, USA) and the results were analysed using FlowJo software (Tree Star, Stanford, CA, USA).

Cells were lysed in buffer containing $50 \mathrm{~mm}$ Tris- $\mathrm{HCl}$ ( $\mathrm{pH} 7.4$ ), $150 \mathrm{~mm} \mathrm{NaCl}, 1 \mathrm{~mm}$ EDTA, 1\% Triton X-100, 1\% proteaseinhibitor cocktail (Nacalai Tesque) and 1\% phosphatase-inhibitor cocktail (Nacalai Tesque). Following centrifugation $\left(14000 \mathrm{~g}, 4^{\circ} \mathrm{C}\right.$, $15 \mathrm{~min}$ ), soluble protein was prepared at $100 \mu \mathrm{g} / 90 \mu \mathrm{l}$. HeparinaseIII $(10 \mathrm{U} / 10 \mu \mathrm{l})$ (Sigma-Aldrich) was added to each aliquot, and digestion was performed for $6 \mathrm{~h}$ at $37^{\circ} \mathrm{C}$. For immune-precipitation, $2 \mu \mathrm{g}$ of anti-GPC1 antibody was added, with incubation on a rotary mixture at $4{ }^{\circ} \mathrm{C}$ for $1 \mathrm{~h}$. A protein $\mathrm{G}$ sepharose (GE healthcare, Little Chalfont, Buckinghamshire, UK) bead slurry $(20 \mu \mathrm{l})$ was then added to capture immune complexes. The mixture was then incubated on a rotary mixer at $4{ }^{\circ} \mathrm{C}$ for $1 \mathrm{~h}$. The slurry was centrifuged at $14500 \mathrm{~g}$ for $30 \mathrm{~s}$ at $4{ }^{\circ} \mathrm{C}$, and the supernatant was discarded. Beads were washed three times with $1 \mathrm{ml}$ of lysis buffer, with centrifugation steps between each wash. To elute the proteins from the beads, $10 \mu \mathrm{l}$ of $2 \times$ SDS-sample buffer (non-reducing conditions) was added to each sample and heated at $95{ }^{\circ} \mathrm{C}$ for $5 \mathrm{~min}$. The supernatant was then collected (Svensson and Mani, 2009; Svensson et al, 2011).

Eluted proteins were resolved by electrophoresis and blotted to PVDF membranes, which were blocked using 5\% BSA (Albumin, Bovine, F-V, Nacalai Tesque) in TBS-T solution as previously described (Souma et al, 2012; Natatsuka et al, 2015). They were incubated overnight at $4{ }^{\circ} \mathrm{C}$ with the anti-human GPC-1 antibody (R\&D Systems Inc.). The samples were then incubated with the donkey anti-goat IgG-HRP (Santa Cruz Biotechnology Inc., Dallas, TX, USA) secondary antibody for $1 \mathrm{~h}$ at room temperature. Visualisation was performed using a Chemi-Lumi One L (Nacalai Tesque).

Measurement of $\mathrm{IC}_{50}$ values after exposure to chemotherapeutic agents. $\mathrm{IC}_{50}$ values were measured after treatment with taxanes (DTX, Sigma-Aldrich), fluoropyrimidine (5-FU, Sigma-Aldrich) and platinum (CDDP, Sigma-Aldrich). Cells cultured in RPMI 1640 medium, supplemented with 10\% FBS without antibiotics, were seeded in 96-well plates. For CDDP exposure, TE-14 and LK- 2 cells were seeded at $2 \times 10^{3}$ cells per well. For exposure to 5FU, TE-14 was seeded at $2 \times 10^{3}$ cells per well and LK-2 at $1 \times 10^{3}$ cells per well. For the DTX experiment, TE-14 and LK-2 cells were both seeded at $1 \times 10^{3}$ cells per well. Cells were cultured for $24 \mathrm{~h}$, and then exposed to various concentrations of DTX (0-20 nM) for $120 \mathrm{~h}, 5-\mathrm{FU}(0-20 \mu \mathrm{M})$ for $96 \mathrm{~h}$ and CDDP $(0-20 \mu \mathrm{M})$ for $72 \mathrm{~h}$. Cellular proliferation was subsequently evaluated by WST- 8 assay, that is, 2-(2-methyosy-4-nitro-phenyl)-3-(4-nitrophenyl)-5-(2, 4disulfophynel)-2H-tetrazolium monosodium salt assay (Cell Counting Kit-SF; Nacalai Tesque). The absorption of WST-8 was measured at $450 \mathrm{~nm}$ (reference wavelength: $630 \mathrm{~nm}$ ) using a Model 680 microplate reader (Bio-Rad Laboratories, Hercules, CA, USA). Absorbance values presented were the average \pm s.d. of triplicate plates for the treated samples, expressed as percentages relative to untreated controls. $\mathrm{IC}_{50}$ values were then calculated by $\mathrm{IC}_{50}=10\{\log (A / B) \times(50-C) /(D-C)+\log (B)\}$, where $A$ represents the higher concentration between the $50 \%$ of the inhibition rate, $B$ represents the lower concentrations between the $50 \%$ of inhibition rate, $C$ represents the inhibition rate at $B$ and $D$ represents the inhibition rate at A (Hirayama et al, 2008; Hossain et al, 2010; Arita-Morioka et al, 2015).

Quantitation of platinum binding to genomic DNA. Cells cultured in antibiotic-free RPMI 1640, supplemented with $10 \%$ FBS, were seeded in $100-\mathrm{mm}$ dishes, and cultured for $24 \mathrm{~h}$ before being exposed to $10 \mu \mathrm{M}$ CDDP for $24 \mathrm{~h}$. Genomic DNA was isolated using the FlexiGene DNA Kit (Qiagen) according to the manufacturer's protocol (Trzeciak et al, 2004). DNA quantification was conducted using a Nanodrop1000 (Thermo Fisher Scientific).

Luminescent assay to measure caspase-3/7 activities. Caspase-3 activity was measured using the Caspase-Glo $3 / 7$ assay kit (Promega, Madison, WI, USA), according to the manufacturer's instructions (Takemoto et al, 2015). Briefly, TE-14, GP-C, GN1 and GN2 cells cultured in RPMI 1640 medium, supplemented with $10 \%$ FBS without antibiotics, were seeded in 96-well plates overnight. CDDP $(5 \mu \mathrm{M})$ was added to the cells for $24 \mathrm{~h}$. To measure caspase activity, luminescence RLU values (following a $1-h$ incubation with the reagent) were measured using a luminometer (GEMINI EM Microplate Reader (Molecular Devices LLC, Sunnyvale, CA, USA)) with the SOFTMAX PRO v. 5.4.5 data-processing system (Molecular Devices, LLC.). Data were calculated by taking the RLU values for each test assay and dividing by untreated negative control values. The averages \pm s.d. of triplicate wells are presented.

Western blotting for effectors of apoptosis. Cells were lysed in radio-immunoprecipitation assay buffer $(10 \mathrm{~mm}$ Tris- $\mathrm{HCl}, \mathrm{pH} 7.5$, 
$150 \mathrm{~mm} \mathrm{NaCl}, 1 \%$ Nonidet P-40, 0.5\% sodium deoxycholate, $0.1 \%$ SDS, $1 \%$ protease-inhibitor cocktail and $1 \%$ phosphatase-inhibitor cocktail). Following centrifugation $\left(14000 \mathrm{~g}, 4^{\circ} \mathrm{C}, 15 \mathrm{~min}\right)$, soluble proteins were resolved using SDS-PAGE, as previously described (Souma et al, 2012; Natatsuka et al, 2015). The primary antibodies used were as follows: anti-phospho-p44/42 (Thr202/Tyr204, $1: 1000)$, anti-p44/42 (1:1000), anti-phosopho-MEK1/2 (Ser217/ 221, $1: 1000)$, anti-MEK1/2 (1:1000), anti-phosopho-Bad (Ser112, $1: 500)$, anti-Bad (1:1000), anti-phospho-Bcl-2 (Ser70, 1:500), anti-Bcl-2 (1:500, Cell Signaling Technology Inc., Danvers, MA, USA), $\beta$-Actin (Sigma-Aldrich, $1: 2000)$ and anti-GAPDH (Santa Cruz Biotechnology Inc; $1: 2000)$. The secondary antibodies were as follows: anti-rabbit, IgG, HRP-linked antibody (Cell Signaling Technology Inc.), HRP-conjugated donkey anti-rabbit IgG (GE Healthcare) and HRP-conjugated sheep anti-mouse IgG (GE Healthcare). Signals were visualised using a luminol-based chemiluminescence assay kit-Chemi-Lumi One L.

Statistical analyses. Statistical analyses were performed using the JMP Pro 11.2.1 (SAS Institute Inc., Cary, NC, USA). Overall survival (OS) was evaluated using the Kaplan-Meier method, and assessed by the log-rank test. All parameters found to be significant by univariate analyses using the Cox proportional hazard model were included in multivariate survival analysis. $\mathrm{IC}_{50}$ values and $\mathrm{Pt}$ binding to genomic DNA were compared for GN1 and GN2 cell lines, and G56 and G57 transfectants using GP-C and E29 as control cells. Statistical analyses were performed using the Tukey HSD test. $P$-values of $<0.05$ were considered significant.

\section{RESULTS}

Quantitative plasma membrane proteomic analysis of normal oesophageal epithelial and ESCC cell lines. To identify the disease specific antigen of ESCC, we performed quantitative protein expression profiling analysis of normal oesophageal epithelial cells (HEEpiC and Het-1A) and ESCC cell lines (TE-1, TE-6, TE-8, TE-9, TE-10 and TE-14) focused on plasma membrane proteins. Using a biotinylation-based approach for enrichment of plasma membrane protein, and combined with the iTRAQ technology using nano-LC-MS/MS analysis, 1638 proteins were identified. The complete list of all proteins identified is shown in Supplementary Table 1. According to annotations from UniProtKB, 401 proteins (24\% of the identified proteins) were located in the plasma membrane (Figure 1A). Among these, 12 proteins were increased $>2$-fold in at least 4 of 6 ESCC cell lines compared with normal oesophageal epithelial cell lines (Table 1). In accordance with the previous report on ESCC (Matsuda et al, 2014), increased expression of an epithelial cell adhesion molecule was found in TE-1, TE-6, TE-9, TE-10 and TE-14 compared with HEEpiC and Het-1A (Table 1).

Notably, GPC1, a HSPG, was found to have one of the most significant differences in expression between the normal oesophageal epithelial and ESCC cell lines (Table 1).

Confirmatory expression analysis of GPC1 in ESCC. To confirm the altered expression of GPC1 in ESCC cells, western blotting was performed using two normal oesophageal epithelial cell lines (HEEpiC and Het-1A) and nine ESCC cell lines (TE-1, TE-5, TE-6, TE-8, TE-9, TE-10, TE-11, TE-14 and TE-15). We observed that GPC1 protein levels were higher than that of normal oesophageal epithelial cells (HEEpiC and Het-1A) (Figure 1B). Flow cytometry was performed to evaluate the presence of GPC1 protein on the cell surface. Surface expression of GPC1 was hardly detectable in HEEpiC, whereas Het-1A cells demonstrated minimal surface expression of GPC1 (Figure 1C). In contrast, considerably higher levels of GPC1 surface expression were detected in all of the ESCC cell lines (Figure 1C). These data demonstrate that GPC1 is overexpressed in ESCC cells at the protein level consistent with iTRAQ analysis.

IHC analysis of GPC1 expression in ESCC specimens. The expression of GPC1 at the basal layer of normal oesophageal tissue was assigned as 'normal' (the internal reference (control) level). GPC1 immunopositivity was principally localised at the cellular membrane. GPC1 scores were generated as described in the Materials and Methods section. Among the 175 cases, we divided them into 2 equally balanced groups by GPC1 stained score. Seventy six cases (43.4\%) scored more than 4 points (denoted as our high-expression group (HG); Figure 1D). The remaining 99 cases (56.6\%) scored less than 3 points (low-expression group (LG); Figure 1E). The distribution of GPC1 expression is shown in Figure 1F.

Correlation between clinicopathological phenotype and GPC1 expression. Supplementary Table 2 shows the correlation between GPC1 expression in ESCC and various clinicopathological parameters. We could find no correlation between high expression of GPC1 and any clinicopathological parameters, including pathologic (p) T, N and $\mathrm{M}$ stages. However, GPC1 expression significantly correlated with poor OS $(P<0.001$, log-rank test) (Figure 1G).

Prognostic significance of GPC1 expression in oesophageal squamous cell cancer. Associations between various clinicopathological parameters and OS were examined using Cox's proportional hazard model. Univariate analyses showed that age, vessel invasion, pT stage, $\mathrm{pN}$ stage, $\mathrm{pM}$ stage and GPC1 score all correlated with OS. Multivariate analyses identified age, $\mathrm{pT}$ stage, $\mathrm{pM}$ stage and GPC1 score, as significant independent prognostic predictors for OS (Table 2).

GPC1 expression and response to chemotherapy. We then examined the association between histological response and GPC1 expression comparing neoadjuvant chemotherapy and surgical cases. Among 97 patients treated with neoadjuvant chemotherapy (DCF therapy), 48 (49.5\%) were categorised (by IHC) as weak (LG) and 49 (50.5\%) as strong expressers of GPC1 (HG). Among the 48 LG patients, 33 (68.7\%) biopsies revealed a good histological response following therapy. Finally, among the 49 HG patients, $12(24.5 \%)$ showed a similarly good histological response. We therefore concluded that there was a significant negative correlation between histological response and high-GPC1 expression $(P<0.001$; Table 3$)$.

Establishment and corroboration of GPC1 transfected/knockout cells. To clarify the association between GPC1 expression and chemoresistance, we investigated using cell lines in vitro. The TE14 cell line expresses GPC1, whereas the LK-2 cell line is GPC1 negative, as analysed by western blotting and flow cytometry. Using these assays we confirmed that LK-2 G56, G57, TE-14 and GP-C were all positive for GPC1. We also confirmed GPC1negative status for TE-14 GN1, GN2, LK-2 and E29 (Figure 2A-D).

Drug susceptibility assay. To investigate the relationship between GPC1 expression and sensitivity to CDDP, 5-FU and DTX, we used the WST-8 assay (Supplementary Figure 1). $\mathrm{IC}_{50}$ values following exposure to CDDP were derived for GPC1-expressing cells; these were significantly higher than those of GPC1-negative cells. For example, the respective $\mathrm{IC}_{50}$ values for $\mathrm{GP}-\mathrm{C}, \mathrm{GN} 1$ and GN2 were $8.76 \mu \mathrm{M}, 4.38 \mu \mathrm{M}$ and $3.18 \mu \mathrm{M}(P<0.0001, P<0.0001)$, respectively. However, the $\mathrm{IC}_{50}$ values for 5 -FU and DTX were unchanged, irrespective of GPC1 expression (Figure 2E and F). In response to these data, we then focused on the mechanistic role surrounding GPC1 expression and resistance to CDDP action.

Measurement of platinum binding to DNA. To elucidate the mechanism underlying CDDP resistance induced by plasma 
A

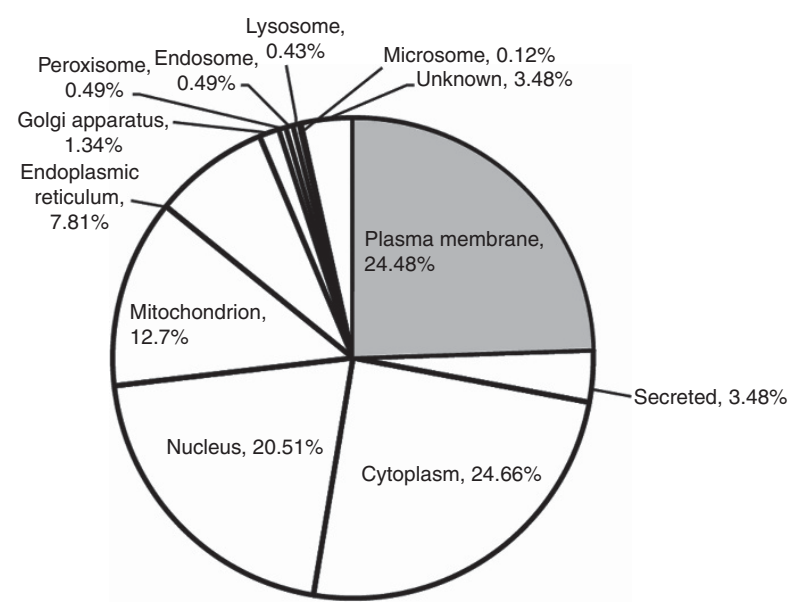

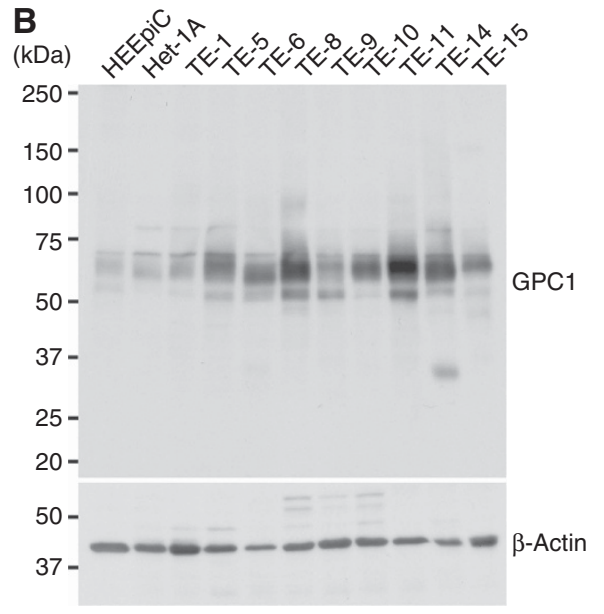

C

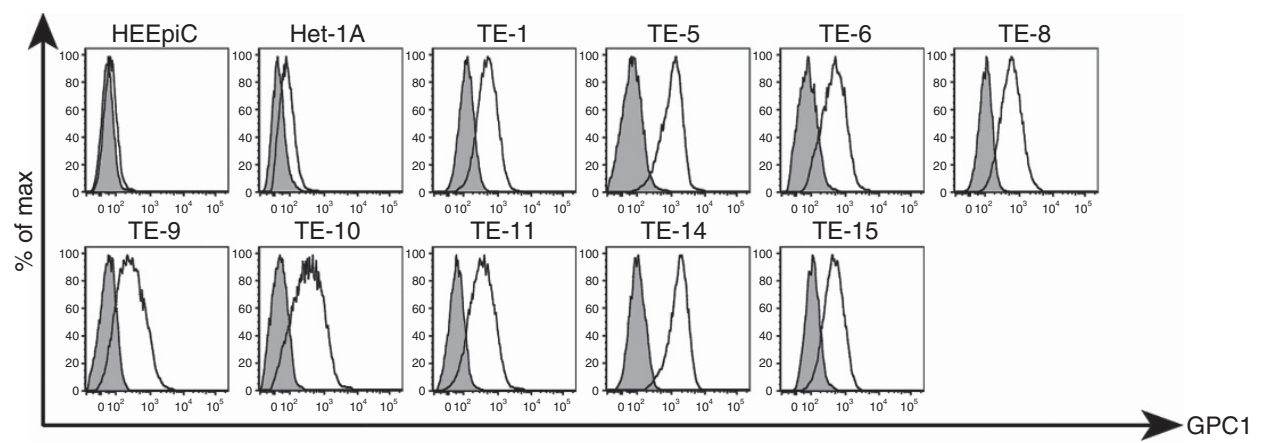

D

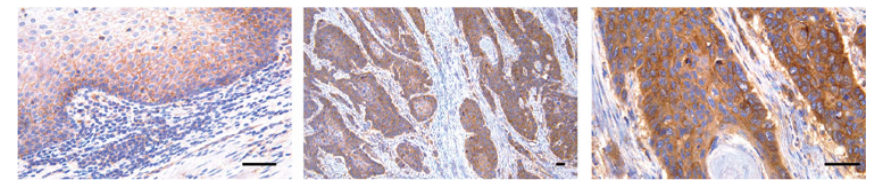

$\mathbf{E}$
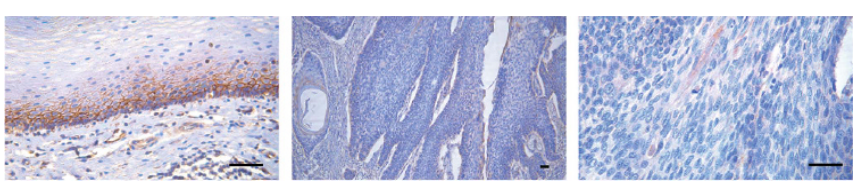

$\mathbf{F}$
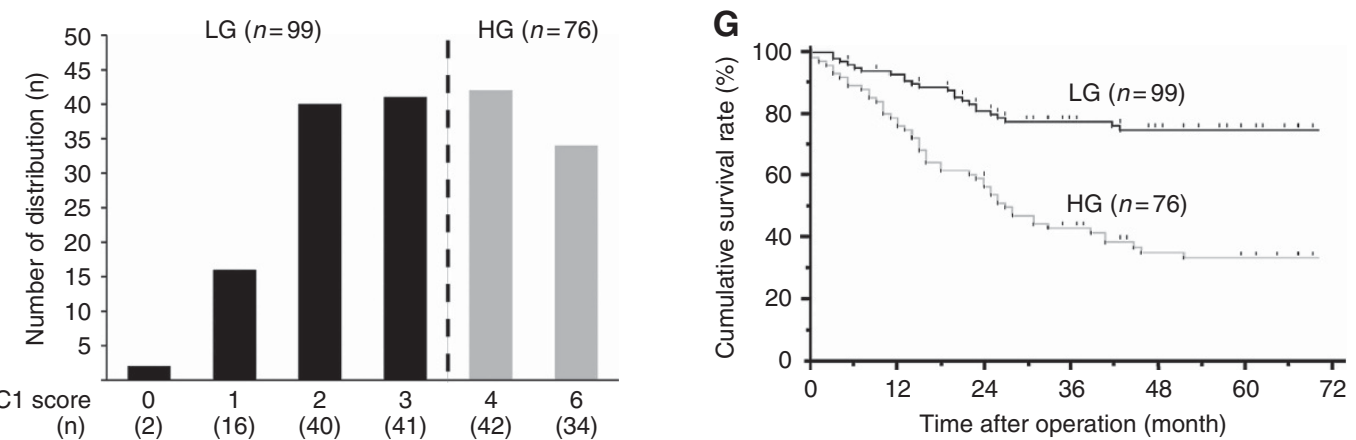

$\begin{array}{llllllll}(\mathrm{n}) & (2) & (16) & (40) & (41) & (42) & (34)\end{array}$

Figure 1. Identification of GPC1 and confirmation by clinicopathological analyses in ESCC. (A) Subcellular localisation of the 1638 identified proteins analysed by UniProtKB. (B) Confirmation of iTRAQ results by western blotting and flow cytometry. Western blotting using the anti-GPC1 antibody against proteins treated with heparinase-III. $\beta$-Actin was used as a loading control. (C) Confirmation of iTRAQ results by flow cytometry. The shaded histogram profile indicates the isotype control and open histogram indicates anti-GPC1 antibody-staining results. Representative immunohistochemical staining for GPC1 in (D) the high-GPC1 expression group (HG) and (E) the low-GPC1 expression group (LG). Left panel: the basal layer of specimen used as an internal control for GPC1 expression in a high-power field; Middle panel: tumour site in a low-power field; Right panel: tumour site in a high-power field. (Scale bar: $50 \mu \mathrm{m}$ ). (F) The distribution of GPC1 scores in LG and HG. (G) Kaplan-Meier analyses of OS. The black line represents $L G$ and the grey line represents $H G$, showing their survival rate during the study observation period. 
Table 1. Plasma membrane proteins overexpressed in oesophageal cancer cells

\section{iTRAQ ratio}

\begin{tabular}{|c|c|c|c|c|c|c|c|c|c|}
\hline Accession number & Protein name & $\begin{array}{l}\text { No. of peptides } \\
\text { used for identification }\end{array}$ & Het1A & TE-1 & TE-6 & TE-8 & TE-9 & TE-10 & TE-14 \\
\hline O14493 & Claudin-4 & 1 & 1.093 & 1.759 & 2.765 & 0.846 & 2.364 & 2.623 & 5.937 \\
\hline O43688 & Lipid phosphate phosphohydrolase 2 & 1 & 0.346 & 1.176 & 4.730 & 0.867 & 5.875 & 2.640 & 3.259 \\
\hline O60669 & Monocarboxylate transporter 2 & 1 & 1.326 & 2.681 & 0.570 & 3.874 & 5.296 & 1.261 & 24.437 \\
\hline P06213 & Insulin receptor & 3 & 0.595 & 2.647 & 2.465 & 1.527 & 3.103 & 2.128 & 5.570 \\
\hline P16422 & Epithelial cell adhesion molecule & 9 & 1.277 & 2.434 & 3.230 & 0.835 & 3.064 & 5.252 & 3.668 \\
\hline P21583 & Kit ligand & 1 & 1.049 & 3.008 & 2.979 & 1.008 & 4.168 & 2.113 & 4.496 \\
\hline P35052 & Glypican-1 & 13 & 1.216 & 1.704 & 2.851 & 1.634 & 2.245 & 2.931 & 4.787 \\
\hline P43003 & Excitatory amino acid transporter 1 & 1 & 0.992 & 10.748 & 5.416 & 0.931 & 5.057 & 8.523 & 0.647 \\
\hline P61088 & Ubiquitin-conjugating enzyme E2 N & 1 & 0.853 & 1.720 & 2.310 & 2.860 & 2.608 & 2.606 & 2.565 \\
\hline Q10589 & Bone marrow stromal antigen 2 & 3 & 1.710 & 2.113 & 7.358 & 2.027 & 5.364 & 7.990 & 1.218 \\
\hline Q12860 & Contactin-1 & 16 & 0.832 & 0.628 & 5.068 & 0.988 & 2.328 & 2.932 & 2.085 \\
\hline Q9Y4D7 & Plexin-D1 & 3 & 1.648 & 3.127 & 1.132 & 3.252 & 3.163 & 1.399 & 2.583 \\
\hline
\end{tabular}

Abbreviation: $\mathrm{ITRAQ}=$ isobaric tag for relative and absolute quantitation. The ITRAQ ratios were calculated considering the oesophageal cancer cells iTRAQ signal divided by the HEEpiC cells ITRAQ signal. Proteins overexpressed more than twofold in at least four cell lines were listed.

Table 2. Univariate and multivariate analyses of overall survival by Cox's proportional hazard model

\begin{tabular}{|c|c|c|c|c|c|c|c|}
\hline Variables & $n$ & \multicolumn{3}{|c|}{ Univariate analysis } & \multicolumn{3}{|c|}{ Multivariate analysis } \\
\hline Gender (male/female) & $155 / 20$ & 1.64 & $0.85-3.69$ & 0.151 & & & \\
\hline pT stage $(T 1,2 / T 3,4)$ & $79 / 96$ & 2.72 & $1.72-4.44$ & 0.0001 & 1.96 & $1.13-3.47$ & 0.0153 \\
\hline pN stage (N0/N1-3) & $61 / 114$ & 2.45 & $1.51-4.20$ & 0.0002 & 1.63 & $0.90-3.09$ & 0.107 \\
\hline Vessel invasion (negative/positive) & $104 / 71$ & 1.88 & $1.23-2.86$ & 0.0035 & 1.34 & $0.80-2.22$ & 0.261 \\
\hline Tissue type (grade 1, 2/3, 4) & $43 / 132$ & 1.19 & $0.52-1.39$ & 0.490 & & & \\
\hline Tumour location (Ut, Mt/Lt, Ae) & $113 / 62$ & 1.46 & $0.94-2.23$ & 0.917 & & & \\
\hline GPC1 expression (low/high) & $99 / 76$ & 3.12 & $1.96-5.10$ & $<0.0001$ & 3.08 & $1.91-5.11$ & $<0.0001$ \\
\hline
\end{tabular}

Table 3. Correlation between GPC1 expression and histological effect of neoadjuvant chemotherapy

\begin{tabular}{|l|c|c|}
\hline Histological effect & GPC1 low $(\boldsymbol{n}=\mathbf{4 8})$ & GPC1 high $(\boldsymbol{n}=\mathbf{4 9})$ \\
\hline Grade 0, 1a $(n=52)$ & 15 & 37 \\
\hline Grade 1b, 2 $(n=45)$ & 33 & 12 \\
\hline Abbreviation: GPC1 = glypican- 1.
\end{tabular}

membrane-expressed GPC1, we first assessed platinum binding to the genomic DNA of TE-14 and LK-2 cell lines. Platinum bound to GP-C, GN1 and GN2, was found to be $6.68 \pm 1.22 \mathrm{pg} \mu \mathrm{l}^{-1}$, $5.83 \pm 0.64 \mathrm{pg}^{-1} \mathrm{l}^{-1}(P=0.58)$ and $6.42 \pm 0.29 \mathrm{pg}^{-1} \mathrm{l}^{-1}(P=0.95)$, respectively. The corresponding amounts for E29, G56 and G57 were $3.76 \pm 0.49 \mathrm{pg}^{-1} \mathrm{l}^{-1}, 3.04 \pm 0.45 \mathrm{pg}^{-1} \mathrm{l}^{-1}(P=0.16)$ and $3.26 \pm 0.23 \mathrm{pg}^{-1} \mathrm{l}^{-1}(P=0.35)$, respectively. Therefore, we could find no significant change in platinum binding, despite altered GPC1 expression (Figure 3A and B).

Evaluation of the mechanism underlying GPC1-mediated chemoresistance to CDDP. We next assessed the activity of downstream fate pathways (i.e., apoptosis) that could be modulated by GPC1 expression. First, we measured levels of caspase-3, using a specific fluorogenic peptide substrate, following exposure to CDDP. As shown in Figure 3C, caspase-3 activation was significantly lower in GPC1-expressing cells. These results indicate that GPC1 was involved in modulating the activation of caspase3-mediated apoptosis.

To further refine the molecular basis of GPC1's effect on CDDP-induced apoptosis, we then analysed TE-14 GPC1 knockouts, comparing these with control cells. Western blotting was used to assess the phosphorylation status (i.e., activity) of several key signalling regulators. These included $\mathrm{p}-\mathrm{MEK} 1 / 2$ (phosphorylated at Ser217/221), MEK1/2, p-p44/42 (Thr202/Tyr204), p44/42 (Figure 3D), p-Bad (Ser112), Bad, p-Bcl-2 (Ser70) and Bcl-2 (Figure $3 \mathrm{E}$ ). We found that the TE-14-GPC1 knockouts showed a notable decrease in p-MEK1/2 (Ser217/221) levels, whereas totalMEK1/2 levels were unchanged, relative to controls. Following exposure to $2 \mu \mathrm{M}$ CDDP, TE-14 cells showed a notable increase in p-MEK1/2 (Ser217/221) and p-p44/42 (Thr202/Tyr204) levels; these changes were not replicated in TE-14-GPC1 knockouts. Furthermore, unlike TE-14-GPC1 knockouts, TE-14 cells showed increased $\mathrm{p}$-Bad (Thr112) and p-Bcl-2 (Ser70) expression after drug exposure, both of which function as anti-apoptotic effectors (Figure 3E). 
A

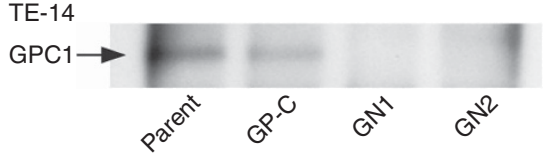

B LK-2

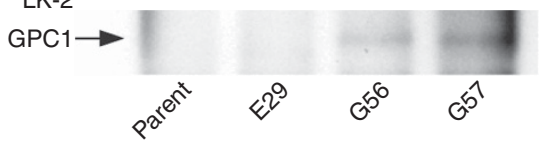

C

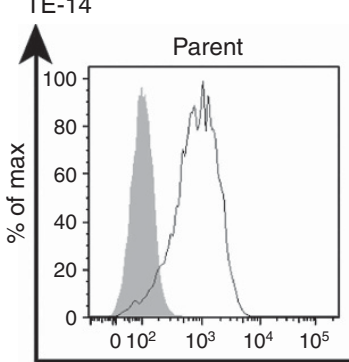

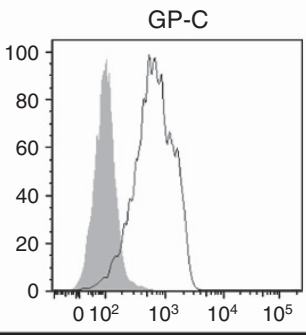
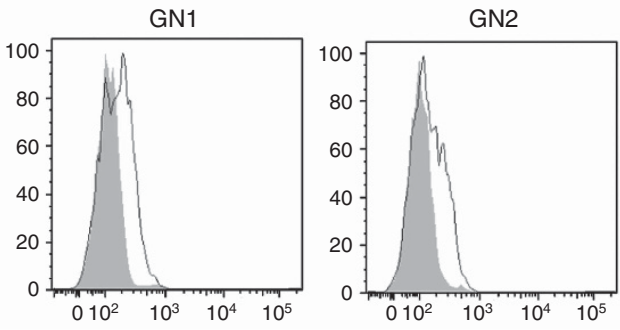

GPC1

D
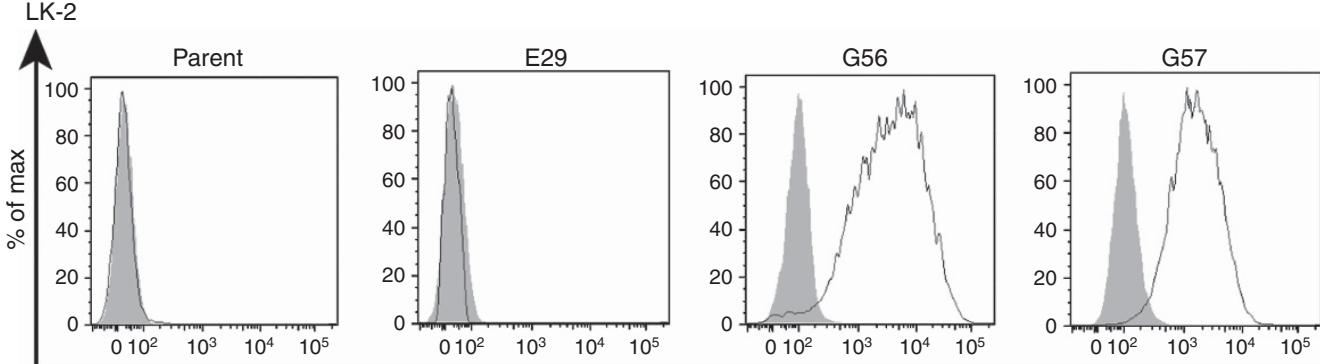

GPC1

E TE-14
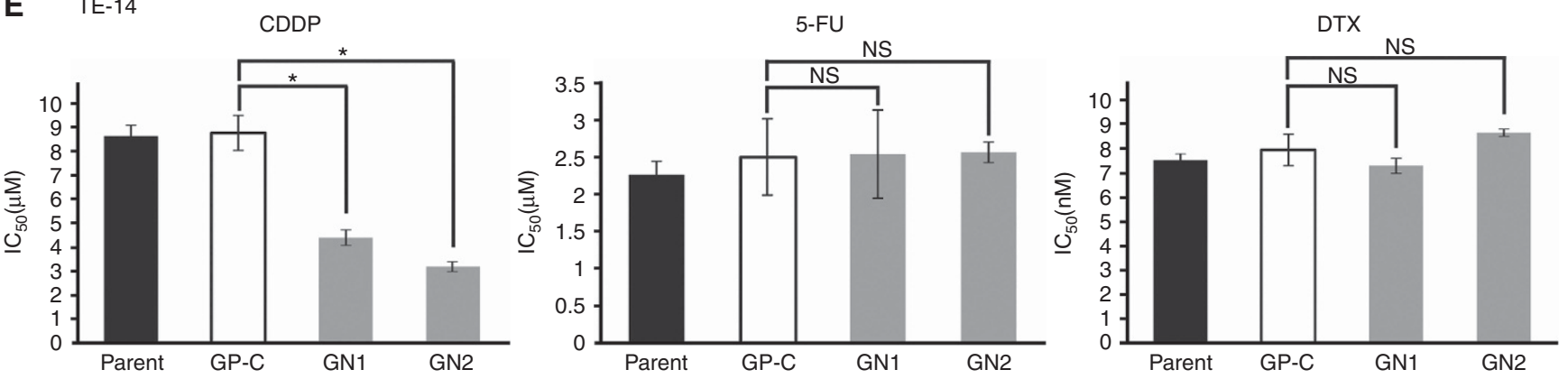

F LK-2
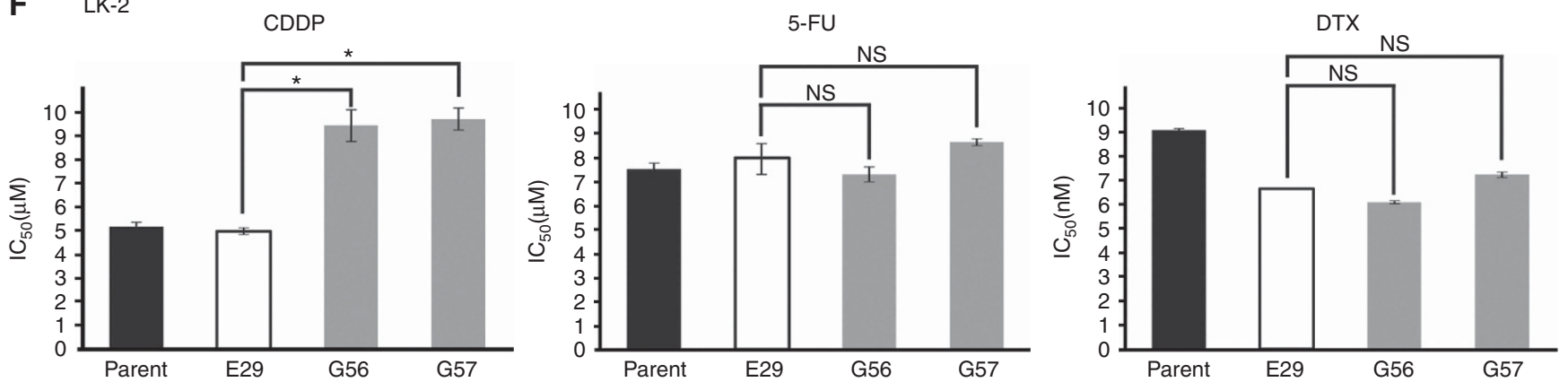

Figure 2. Confirmation of GPC1 status of generated cell lines and their impact of chemo-sensitivity. (A) TE-14, left to right, parental cell line, GPC, GN1 and GN2. The arrow indicates GPC1 expression. (B) LK-2, left to right, parental cell line, E29, G56 and G57. The arrow indicates GPC1 expression. (C) GPC1 expression analyses using flow cytometry in GPC1-knockout (TE-14) cell lines. Grey histograms indicate staining with control IgG, with white histograms showing staining achieved using an anti-CPC1 reagent. Lanes (left to right) indicate parental cell line, GP-C, GN1 and GN2. (D) Similar flow cytometric analyses of GPC1 in LK-2. Lanes (left to right) indicate parental cell line, E29, G56 and G57. (E) Drug susceptibility assay using the WST-8 assay. IC 50 values are shown for each TE-14-derived cell line for the following drug treatments. Left, CDDP ( $\mu \mathrm{m})$; middle, 5 FU $(\mu \mathrm{M})$; right, DTX (nM). NS denotes not significant, ${ }^{\star} P<0.05$. $(F) I C_{50}$ values for $L K-2$-derived cell lines. 
A

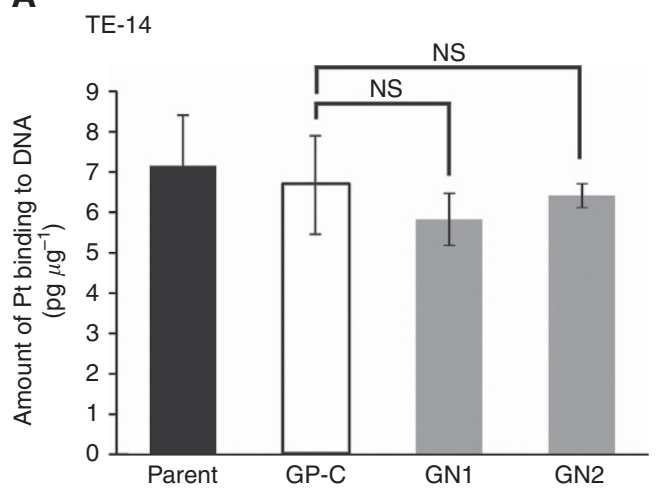

B

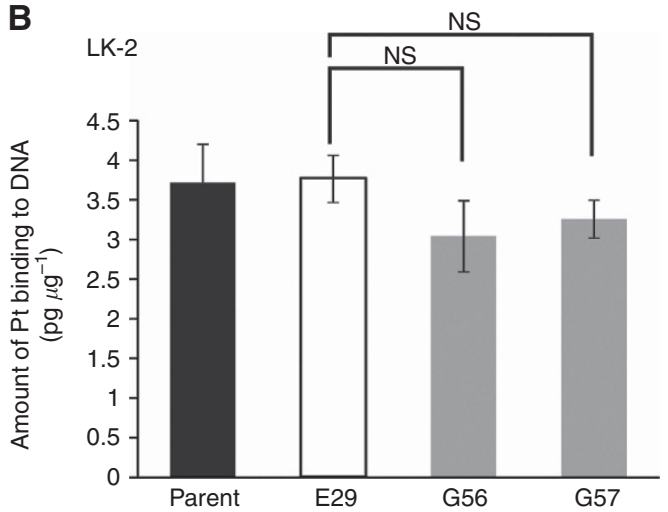

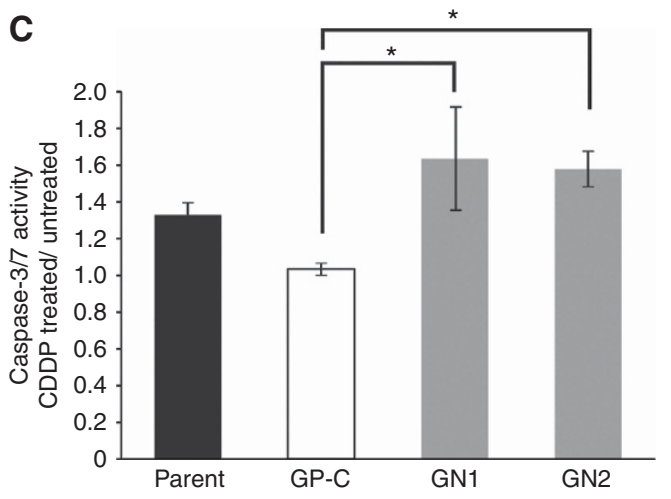

D

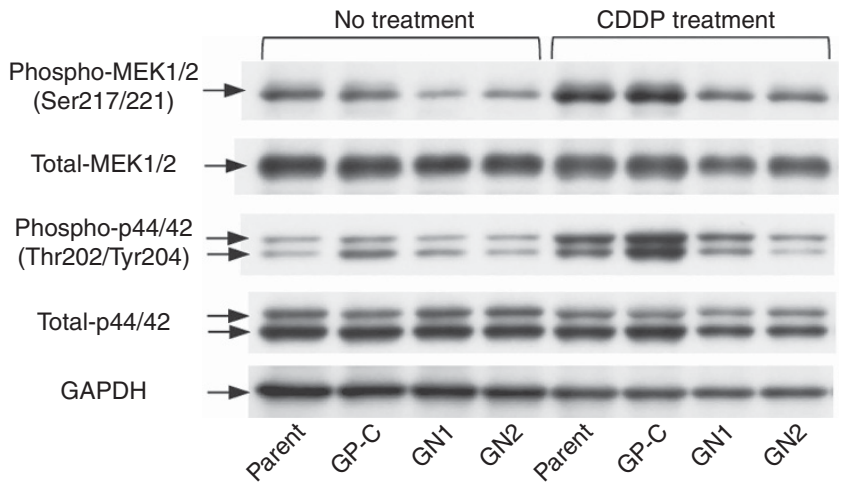

$E$

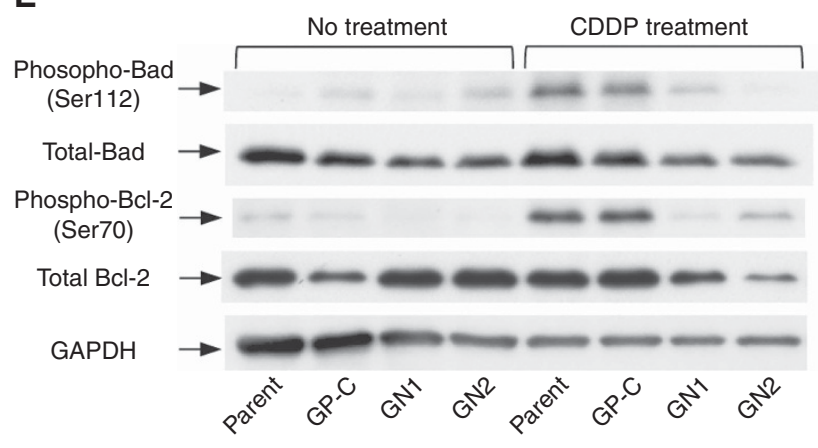

Figure 3. Investigation for the mechanism of GPC1 mediated drug resistance to CDDP. (A) Pt binding to DNA (pg $\mu \mathrm{g}^{-1}$ ) did not significantly differ between GP-C, GN1 and GN2 or (B) between E29, G56 and G57. (C) Caspase-3 activity in TE-14 was measured by luminescent assay. Our data show cell lines treated with $5 \mu \mathrm{m}$ for $24 \mathrm{~h}$ together with untreated controls. (D) MAPK signalling in TE-14 cell lines either untreated (four lanes to the left) or treated with $2 \mu \mathrm{M}$ CDDP for $48 \mathrm{~h}$ (right-hand side lanes). Panels indicate, from top to bottom, phospho-MEK1/2 (Ser217/221), totalMEK1/2, phospho-p44/42 (Thr202/Tyr204), total-p44/42 and GAPDH as a loading control. (E) Bcl-2 family expression in the TE-14 cell lines. As mentioned, only the four lanes to the right were exposed to $2 \mu \mathrm{M}$ CDDP, for $48 \mathrm{~h}$. Panels from top to bottom indicate phospho-Bad (Ser112), totalBad, phospho-Bcl-2 (Ser70), total-Bcl-2 and GAPDH as a loading control. NS denotes not significant, * $P<0.05$.

\section{DISCUSSION}

GPC1 is a member of the glypican family, which comprises HSPGs, linked via a glycosyl-phosphatidylinositol (GPI) anchor to the extracellular membrane. GPC1 functions in angiogenesis, cell-cycle promotion and mitogenic growth in cancer cells (Qiao et al, 2008, 2012, 2013). Other reports have shown that GPC1 is essential for mitogenic signalling promoted by FGF2 and HB-EGF, and plays a crucial role in neoplastic transformation and tumour progression in some cancers (Kleeff et al, 1998, 1999; Gengrinovitch et al, 1999; Matsuda et al, 2001; Ding et al, 2005; Su et al, 2006; Aikawa et al, 2008; Okolicsanyi et al, 2014). It has also been reported that $56 \%$ of clinical samples express GPC 1 in pancreatic cancer, making this protein a possible prognostic marker (Duan et al, 2013). In this study, we identified GPC1 expression in ESCC cell lines, and our IHC analyses showed that 98.8\% of ESCC specimens were GPC1 positive, with expression primarily at the cell membrane. Although there were no significant associations between GPC1 overexpression and clinicopathological factors, such as $\mathrm{pT}$ stage, $\mathrm{pN}$ stage and tissue type, GPC1 overexpression was found to be an independent prognostic factor for OS (by multivariate analyses).

We examined the association between GPC1 overexpression and the histopathological response to neoadjuvant chemotherapy. Our clinical results showed that GPC1 overexpression was significantly associated with resistance to chemotherapy (Table 3). Therefore, we tried to clarify the mechanism of 
the association in vitro. Although the sensitivity to CDDP seen in TE-14-GPC1 knock out cells increased relative to the control cell line, resistance to 5-FU and DTX remained unchanged. Conversely, enhanced chemoresistance to CDDP was found in LK-2 cells forced to express GPC1 compared with a mock GPC1 transfectant as a control. Collectively, these data indicate that the expression of GPC1 may be involved specifically in CDDP resistance. To clarify the mechanism of CDDP resistance, we then evaluated platinum binding to DNA. Our results showed no effect of GPC1 expression on platinum binding. These data suggest no alteration in drug availability, and instead implicate GPC1 action at a stream effector. We therefore examined any change of activity in p-MEK1/2 (Ser217/221) and p-p44/42 (Thr202/Tyr204) kinases (Galluzzi et al, 2012). The TE-14 cell line showed a notable increase in p-MEK1/2(Ser217/221) and p-p44/42 (Thr202/Tyr204), which was not found in TE-14-GPC1 knockouts. The activity of this MAPK pathway is relevant to the regulation of apoptosis, specifically Bcl-2 and Bad. A change in chemo-sensitivity may be due to alterations in apoptotic effector activity. Inhibition of apoptosis mediated by Bcl-2 and Bad phosphorylation, in particular, may partially be responsible for this observed change in chemo-sensitivity.

In this study, we attempted to evaluate the relationship between pre-chemotherapy biopsy material and chemotherapeutic response. Although we examined endoscopic biopsy samples prior to chemotherapy (data not shown), these samples were too small to perform an accurate evaluation of GPC1 expression compared with expression at the basal layer (used as our internal control). Therefore, we used surgical samples post chemotherapy to retrospectively evaluate the relationship between GPC1 expression and chemotherapeutic response. Recently, it has been reported that GPC1 could be detected in circulating exosomes derived from pancreatic cancer. We believe that the GPC1 identification could, therefore, serve as a potential non-invasive diagnostic tool for early-stage pancreatic cancer (Melo et al, 2015). Here, we showed that GPC1 is also highly expressed in ESCC, and may also serve as a biomarker for this lesion. Consequently, we are now attempting to establish new methodologies with which to capture and quantitate GPC1 positive exosomes, as well as serum soluble GPC1. These methods could then be used in clinical diagnoses, and help us to select the most appropriate chemotherapeutic agent, taking into account the chemo-sensitivity modulating the action of GPC1. Our results indicate that GPC1 might also be useful for diagnosis of ESCC.

In conclusion, GPC1 is shown to be an independent prognostic factor for OS in ESCC. In addition, this protein appears to be critical in mediating chemoresistance to CDDP. Taken together, these results suggest that inhibition of overexpressed GPC1 will be a promising therapeutic strategy for patients with advanced ESCC.

\section{ACKNOWLEDGEMENTS}

We would like to thank Y. Kanazawa, J. Yamagishi, M. Yamamoto and Y. Matsuhisa for their secretarial assistance. This research was supported by the Research on Development of New Drugs from Japan Agency for Medical Research and Development, AMED (15ak0101024h0002). We would also like to thank Editage (www.editage.jp) for language editing.

\section{CONFLICT OF INTEREST}

The authors declare no conflict of interest.

\section{REFERENCES}

Aikawa T, Whipple CA, Lopez ME, Gunn J, Young A, Lander AD, Korc M (2008) Glypican-1 modulates the angiogenic and metastatic potential of human and mouse cancer cells. J Clin Invest 118(1): 89-99.

Arita-Morioka K, Yamanaka K, Mizunoe Y, Ogura T, Sugimoto S (2015) Novel strategy for biofilm inhibition by using small molecules targeting molecular chaperone DnaK. Antimicrob Agents Chemother 59(1): 633-641.

Ding K, Lopez-Burks M, Sanchez-Duran JA, Korc M, Lander AD (2005) Growth factor-induced shedding of syndecan-1 confers glypican-1 dependence on mitogenic responses of cancer cells. J Cell Biol 171(4): 729-738.

Duan L, Hu XQ, Feng DY, Lei SY, Hu GH (2013) GPC-1 may serve as a predictor of perineural invasion and a prognosticator of survival in pancreatic cancer. Asian J Surg 36(1): 7-12.

Ferlay J, Soerjomataram I, Dikshit R, Eser S, Mathers C, Rebelo M, Parkin DM, Forman D, Bray F (2015) Cancer incidence and mortality worldwide: sources, methods and major patterns in GLOBOCAN 2012. Int J Cancer 136(5): E359-E386.

Fujita H (2015) History of lymphadenectomy for oesophageal cancer and the future prospects for oesophageal cancer surgery. Surg Today 45(2): 140-149.

Galluzzi L, Senovilla L, Vitale I, Michels J, Martins I, Kepp O, Castedo M, Kroemer G (2012) Molecular mechanisms of cisplatin resistance. Oncogene 31(15): 1869-1883.

Gengrinovitch S, Berman B, David G, Witte L, Neufeld G, Ron D (1999) Glypican-1 Is a VEGF165 binding proteoglycan that acts as an extracellular chaperone for VEGF165. J Biol Chem 274(16): 10816-10822.

Hewitt SM, Baskin DG, Frevert CW, Stahl WL, Rosa-Molinar E (2014) Controls for immunohistochemistry: the Histochemical Society's standards of practice for validation of immunohistochemical assays. $J$ Histochem Chem 62(10): 693-697.

Hirao M, Ando N, Tsujinaka T, Udagawa H, Yano M, Yamana H, Nagai K, Mizusawa J, Nakamura K (2011) Influence of preoperative chemotherapy for advanced thoracic oesophageal squamous cell carcinoma on perioperative complications. Br J Surg 98(12): 1735-1741.

Hirayama C, Watanabe H, Nakashima R, Nanbu T, Hamada A, Kuniyasu A, Nakayama H, Kawaguchi T, Saito H (2008) Constitutive overexpression of $\mathrm{P}$-glycoprotein, rather than breast cancer resistance protein or organic cation transporter 1, contributes to acquisition of imatinib-resistance in K562 cells. Pharmaceut Res 25(4): 827-835.

Hossain MM, Hosono-Fukao T, Tang R, Sugaya N, van Kuppevelt TH, Jenniskens GJ, Kimata K, Rosen SD, Uchimura K (2010) Direct detection of HSulf-1 and HSulf-2 activities on extracellular heparan sulphate and their inhibition by PI-88. Glycobiology 20(2): 175-186.

Japanese Gastric Cancer Association (2011) Japanese classification of gastric carcinoma: 3rd English edition. Gastric Cancer 14(2): 101-112.

Karimata H, Shimoji H, Nishimaki T (2015) Clinicopathological factors predicting R0 resection and long-term survival after esophagectomy in patients with T4 oesophageal cancer undergoing induction chemotherapy or chemoradiotherapy. Surg Today 45(4): 479-486.

Kleeff J, Ishiwata T, Kumbasar A, Friess H, Buchler MW, Lander AD, Korc M (1998) The cell-surface heparan sulphate proteoglycan glypican-1 regulates growth factor action in pancreatic carcinoma cells and is overexpressed in human pancreatic cancer. J Clin Invest 102(9): $1662-1673$.

Kleeff J, Wildi S, Kumbasar A, Friess H, Lander AD, Korc M (1999) Stable transfection of a glypican-1 antisense construct decreases tumorigenicity in PANC-1 pancreatic carcinoma cells. Pancreas 19(3): 281-288.

Matsuda K, Maruyama H, Guo F, Kleeff J, Itakura J, Matsumoto Y, Lander AD, Korc M (2001) Glypican-1 is overexpressed in human breast cancer and modulates the mitogenic effects of multiple heparin-binding growth factors in breast cancer cells. Cancer Res 61(14): 5562-5569.

Matsuda T, Takeuchi H, Matsuda S, Hiraiwa K, Miyasho T, Okamoto M, Kawasako K, Nakamura R, Takahashi T, Wada N, Kawakubo H, Saikawa Y, Omori T, Kitagawa Y (2014) EpCAM, a potential therapeutic target for oesophageal squamous cell carcinoma. Ann Surg Oncol 21 Suppl 3: S356-S364.

Melo SA, Luecke LB, Kahlert C, Fernandez AF, Gammon ST, Kaye J, LeBleu VS, Mittendorf EA, Weitz J, Rahbari N, Reissfelder C, Pilarsky C, Fraga 
MF, Piwnica-Worms D, Kalluri R (2015) Glypican-1 identifies cancer exosomes and detects early pancreatic cancer. Nature 523(7559): 177-182.

Natatsuka R, Takahashi T, Serada S, Fujimoto M, Ookawara T, Nishida T, Hara H, Nishigaki T, Harada E, Murakami T, Miyazaki Y, Makino T, Kurokawa Y, Yamasaki M, Miyata H, Nakajima K, Takiguchi S, Kishimoto T, Mori M, Doki Y, Naka T (2015) Gene therapy with SOCS1 for gastric cancer induces G2/M arrest and has an antitumour effect on peritoneal carcinomatosis. Br J Cancer 113(3): 433-442.

Okolicsanyi RK, van Wijnen AJ, Cool SM, Stein GS, Griffiths LR, Haupt LM (2014) Heparan sulphate proteoglycans and human breast cancer epithelial cell tumorigenicity. J Cell Biochem 115(5): 967-976.

Okumura H, Uchikado Y, Setoyama T, Matsumoto M, Owaki T, Ishigami S, Natsugoe S (2014) Biomarkers for predicting the response of oesophageal squamous cell carcinoma to neoadjuvant chemoradiation therapy. Surg Today 44(3): 421-428.

Pennathur A, Gibson MK, Jobe BA, Luketich JD (2013) Oesophageal carcinoma. Lancet 381(9864): 400-412.

Qiao D, Meyer K, Friedl A (2012) Glypican-1 stimulates Skp2 autoinduction loop and G1/S transition in endothelial cells. J Biol Chem 287(8): 5898-5909.

Qiao D, Meyer K, Friedl A (2013) Glypican 1 stimulates S phase entry and DNA replication in human glioma cells and normal astrocytes. Mol Cell Biol 33(22): 4408-4421.

Qiao D, Yang X, Meyer K, Friedl A (2008) Glypican-1 regulates anaphase promoting complex/cyclosome substrates and cell cycle progression in endothelial cells. Mol Biol Cell 19(7): 2789-2801.

Ross PL, Huang YN, Marchese JN, Williamson B, Parker K, Hattan S, Khainovski N, Pillai S, Dey S, Daniels S, Purkayastha S, Juhasz P, Martin S, Bartlet-Jones M, He F, Jacobson A, Pappin DJ (2004) Multiplexed protein quantitation in Saccharomyces cerevisiae using amine-reactive isobaric tagging reagents. Mol Cell Proteomics 3(12): 1154-1169.

Rustgi AK, El-Serag HB (2014) Oesophageal carcinoma. N Engl J Med 371(26): 2499-2509.

Souma Y, Nishida T, Serada S, Iwahori K, Takahashi T, Fujimoto M, Ripley B, Nakajima K, Miyazaki Y, Mori M, Doki Y, Sawa Y, Naka T (2012)
Antiproliferative effect of SOCS-1 through the suppression of STAT3 and p38 MAPK activation in gastric cancer cells. Int J Cancer 131(6): 1287-1296.

Su G, Meyer K, Nandini CD, Qiao D, Salamat S, Friedl A (2006) Glypican-1 is frequently overexpressed in human gliomas and enhances FGF-2 signaling in glioma cells. Am J Pathol 168(6): 2014-2026.

Svensson G, Hyrenius Wittsten A, Linse S, Mani K (2011) The structural role of N-linked glycans on human glypican-1. Biochemistry 50(43): 9377-9387.

Svensson G, Mani K (2009) S-Nitrosylation of secreted recombinant human glypican-1. Glycoconj J 26(9): 1247-1257.

Takemoto N, Serada S, Fujimoto M, Honda H, Ohkawara T, Takahashi T, Nomura S, Inohara H, Naka T (2015) Leucine-rich alpha-2-glycoprotein promotes TGFbetal-mediated growth suppression in the Lewis lung carcinoma cell lines. Oncotarget 6(13): 11009-11022.

Trzeciak AR, Nyaga SG, Jaruga P, Lohani A, Dizdaroglu M, Evans MK (2004) Cellular repair of oxidatively induced DNA base lesions is defective in prostate cancer cell lines, PC-3 and DU-145. Carcinogenesis 25(8): 1359-1370.

Yokoyama T, Enomoto T, Serada S, Morimoto A, Matsuzaki S, Ueda Y, Yoshino K, Fujita M, Kyo S, Iwahori K, Fujimoto M, Kimura T, Naka T (2013) Plasma membrane proteomics identifies bone marrow stromal antigen 2 as a potential therapeutic target in endometrial cancer. Int J Cancer 132(2): 472-484.

Zheng Y, Li Y, Liu X, Sun H, Wang Z, Zhang R (2015) Reevaluation of neoadjuvant chemotherapy for oesophageal squamous cell carcinoma: a meta-analysis of randomized controlled trials over the past 20 years. Medicine (Baltimore) 94(27): e1102.

This work is published under the standard license to publish agreement. After 12 months the work will become freely available and the license terms will switch to a Creative Commons AttributionNonCommercial-Share Alike 4.0 Unported License.

Supplementary Information accompanies this paper on British Journal of Cancer website (http://www.nature.com/bjc) 